\title{
Marked Arteriosclerosis of the Descending Aorta in a Teenager with Interruption of the Aorta
}

\author{
Sachio Kawai, Ryozo Okada, Hidehiko Sakurai, Hiroshi Yamaguchi and Yoshiro Fukuda*
}

\begin{abstract}
Marked arteriosclerosis localized to the descending thoracic and abdominal aorta was observed in a 19-year-old girl with interruption of the aorta (type $A$ ) and a ventricular septal defect. She died from rupture of saccular aneurysm of the pulmonary trunk. The descending thoracic and abdominal aorta showed marked thrombogenic, lamellar thickening of the intima. In contrast, no significant arteriosclerosis was detected in the ascending aorta. Several factors including diffuse sludging of blood, mural thrombosis and hemodynamic changes, in close association with polycythemia, are hypothesized to be causative factors in the development of this lesion.
\end{abstract}

(Internal Medicine 35: 383-387, 1996)

Key words: congenital heart disease, pulmonary aneurysm, rupture, cardiac tamponade

\section{Introduction}

Almost all patients with aortic arch interruption die within the first month of life unless treated surgically (1) and rarely reach adulthood (2). In aortic arch interruption, the descending aorta is supplied by mixed venous blood with relatively low pressure through the ductus arteriosus following birth. We have encountered a 19-year-old patient with aortic arch interruption whose descending aorta showed unique pathologic changes.

\section{Case Report}

This is a case of a 19-year-old girl who was born of a normal delivery. However, her mother had cardia valvular disease and died at 40 years of age. A previous child had died at birth due to a cardiac anomaly.

The patient was diagnosed as having cardiac disease soon after birth. In primary school, she had suffered from several episodes of bloody sputum, stridor, dyspnea on effort and marked cyanosis of the lower part of the body (differential cyanosis). At 10 years of age, cardiac catheterization and angiography performed at our hospital (November 1971) demonstrated interruption of the aorta between the left subclavian artery and the ductus arteriosus (type A) (3), a ventricular septal defect with bidirectional shunt (left to right, $3.0 \mathrm{l} / \mathrm{min}$; right to left, $2.0 \mathrm{l} / \mathrm{min}$ ) and pulmonary hypertension $(90 / 70 \mathrm{mmHg})$. Oxygen contents were as follows: $10.7 \mathrm{Vol} \%$ in the superior vena cava, $14.9 \mathrm{Vol} \%$ in the inferior vena cava, $15.1 \mathrm{Vol} \%$ in the right atrium, $20.8 \mathrm{Vol} \%$ in the right ventricle, $19.9 \mathrm{Vol} \%$ in the pulmonary trunk, $22.8 \mathrm{Vol} \%$ in the left ventricle, $21.1 \mathrm{Vol} \%$ in the brachial artery and $19.0 \mathrm{Vol} \%$ in the femoral artery. The pressure in the right ventricle was $116 / 5 \mathrm{mmHg}$, and her blood pressure was $108 / 60 \mathrm{mmHg}$. Since pulmonary hypertension had been established, surgical intervention was not performed. At 13 years of age, she was readmitted briefly due to epistasis. Her hematologic and chemical data were as follows: erythrocyte count, $533 \times 10^{4} / \mathrm{cu} \mathrm{mm}$; hemoglobin, $17.0 \mathrm{~g} / 100 \mathrm{ml}$; hematocrit, $51.2 \%$; leukocyte count, $6,700 / \mathrm{mm}^{3}$; platelet count, $9.5 \times 10^{4} / \mathrm{mm}^{3}$; bleeding time, 8 minutes; Lee-White clotting time, 7 minutes; prothrombin time, 12.9 seconds (control, 13.8 seconds); partial thromboplastin time, 142 seconds (control, 135.3 seconds); and total cholesterol, $169 \mathrm{mg} / 100 \mathrm{ml}$. A serological test for syphilis was nonreactive. In September 1974, the arterial partial pressures and saturation of oxygen were $62.9 \mathrm{mmHg}$ and $93 \%$ in the brachial artery and 46.8 $\mathrm{mmHg}$ and $84.6 \%$ in the femoral artery, respectively. During the next 6 years, she had suffered occasional dyspnea, but she was able to perform daily activities under strict exercise restriction. In May 1980, she developed a cough and hemoptysis. Three days later, she was admitted to our hospital because of severe pain in the chest, back and shoulders.

On physical examination, she appeared poorly developed (35 kg in weight and $152 \mathrm{~cm}$ in height). Marked cyanosis was observed involving her lips and nails, and her fingers and toes were clubbed. She had no arachnodactyly. Her pulse was regular at 108 beat per minute. The blood pressure in her arms

From the Departments of Cardiology and *Pathology, Juntendo University, School of Medicine, Tokyo

Received for publication August 3, 1995; Accepted for publication February 26, 1996

Reprint requests should be addressed to Dr. Sachio Kawai, Department of Cardiology, Juntendo University, School of Medicine, 2-1-1 Hongo, Bunkyo-ku, Tokyo 113 
was $150 / 70 \mathrm{mmHg}$ and that in her legs $140 / 68 \mathrm{mmHg}$. There was no laterality of the pressure. The pulmonary component of the second heart sound ausculating in her pulmonary area was prominent. A basal pansystolic murmur due to ventricular septal defect (Levine V/VI) and a diastolic pulmonary regurgitant murmur (Levine III/VI) accompanied by a thrill were also detected. No continuous murmur was audible. Her liver was

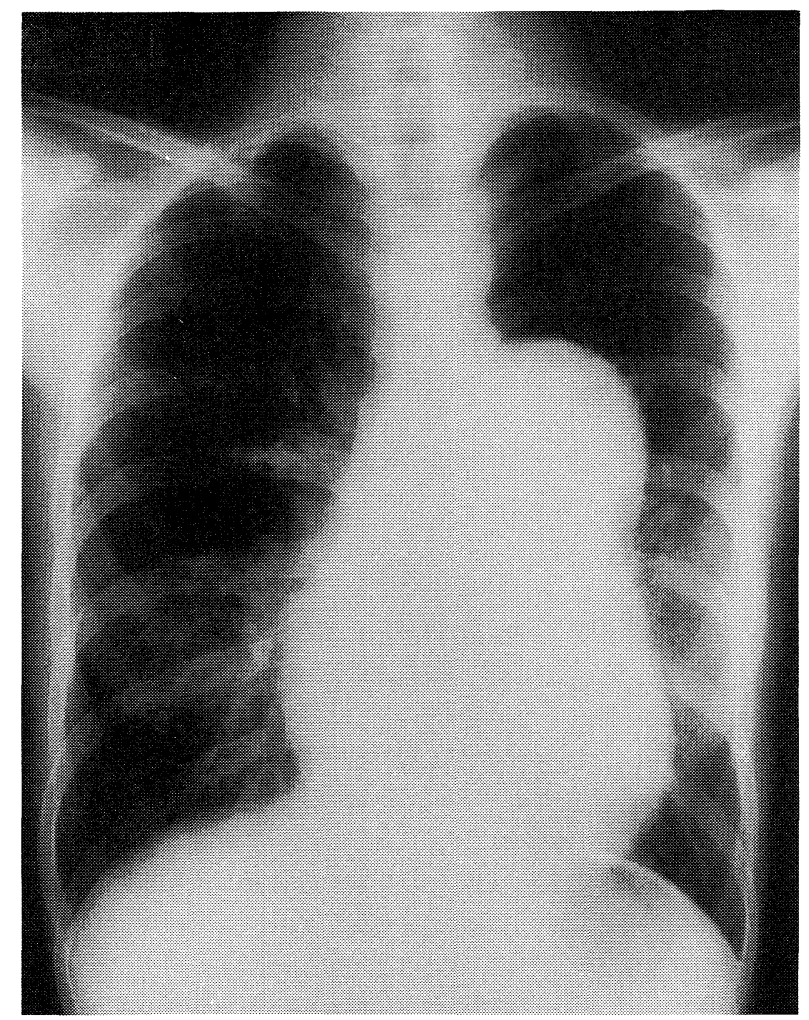

a

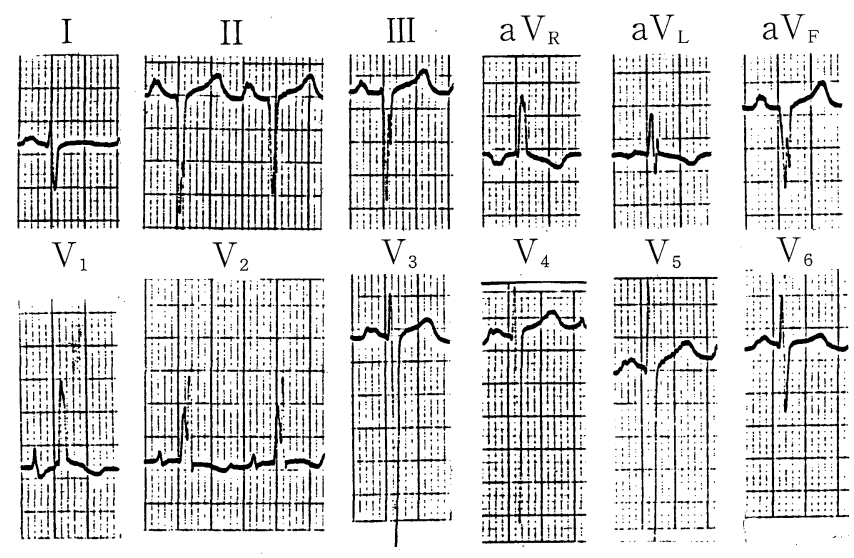

$\mathrm{b}$

Figure 1. Chest radiograph and electrocardiogram. a) Chest radiograph (postero-anterior view) on admission revealing no aortic knob and the prominence of the pulmonary trunk. b) Electrocardiogram on admission demonstrating right ventricular hypertrophy. enlarged and palpable $3 \mathrm{cms}$ below the right costal margin. Edema was not observed in her lower extremities. A chest X-ray film revealed the absence of an aortic knob and prominent dilatation of the pulmonary artery. The cardiothoracic ratio was 57\% (Fig. 1a). An electrocardiogram demonstrated right ventricular hypertrophy (Fig. 1b). One hour following admission, she complained of a recurrence of the severe chest pain and died suddenly. An echocardiographic study was not performed.

\section{Pathological Findings}

The pericardial cavity was distended with $500 \mathrm{ml}$ fresh blood

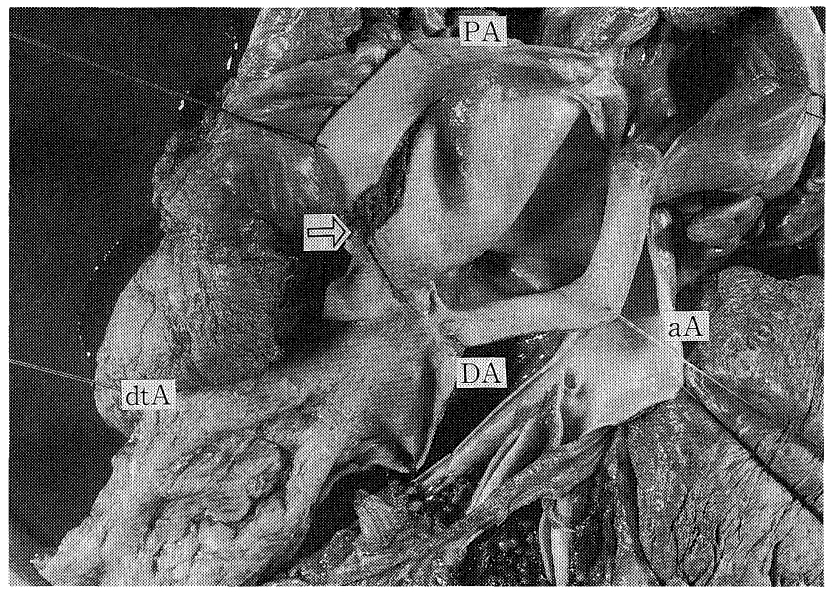

a

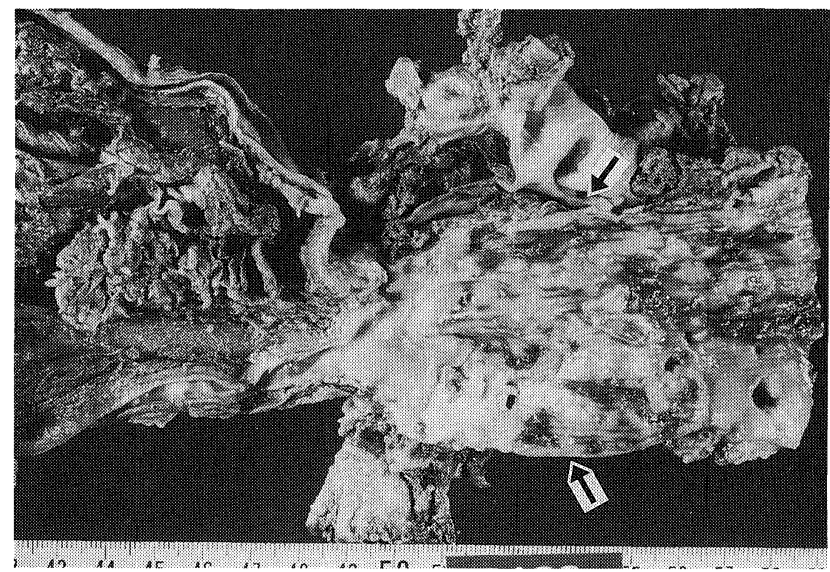

$\mathrm{b}$

Figure 2. Macroscopic findings of the aorta. a) The pulmonary artery, ascending aorta and descending thoracic aorta. The ascending aorta revealed no macroscopic atherosclerotic lesions. Arrows indicate a tear in the main pulmonary artery. The descending thoracic aorta demonstrated marked arteriosclerotic changes except for the area $3 \mathrm{~cm}$ below the insertion to the ductus. aA: ascending aorta, DA: ductus arteriosus, dtA: descending thoracic aorta, PA: pulmonary artery. b) The abdominal aorta and its branches. The abdominal aorta demonstrated marked wavy wrinkled appearance with mural thrombi. The arrows indicate the descending aorta. 
originating from a $2.8 \mathrm{~cm}$ linear tear in the main pulmonary trunk. The heart weighed $280 \mathrm{~g}$. The right ventricle was markedly hypertrophied (thickness of the compact layer was $7.8 \mathrm{~mm}$ in the inflow tract and $10.8 \mathrm{~mm}$ in the outflow tract). A ventricular septal defect $(7.2 \times 15.8 \mathrm{~mm}$ on the right ventricular side and $8.6 \times 15 \mathrm{~mm}$ on the left ventricular side) was observed in the membranous portion. The root of the pulmonary artery was $20 \mathrm{~mm}$ in diameter and the main pulmonary artery was dilated to $40 \mathrm{~mm}$ in diameter. There was no subaortic obstruction. A persistent ductus arteriosus connected the main pulmonary artery and the descending aorta ( $11 \mathrm{~mm}$ in diameter). The aortic root measured $16 \mathrm{~mm}$ in diameter. The inner surface of the ascending artery was smooth and revealed no atherosclerosis. In contrast, marked thickening with a wrinkled appearance was observed in the descending thoracic aorta and abdominal aorta (Fig. 2a and 2b). The circumference of the descending

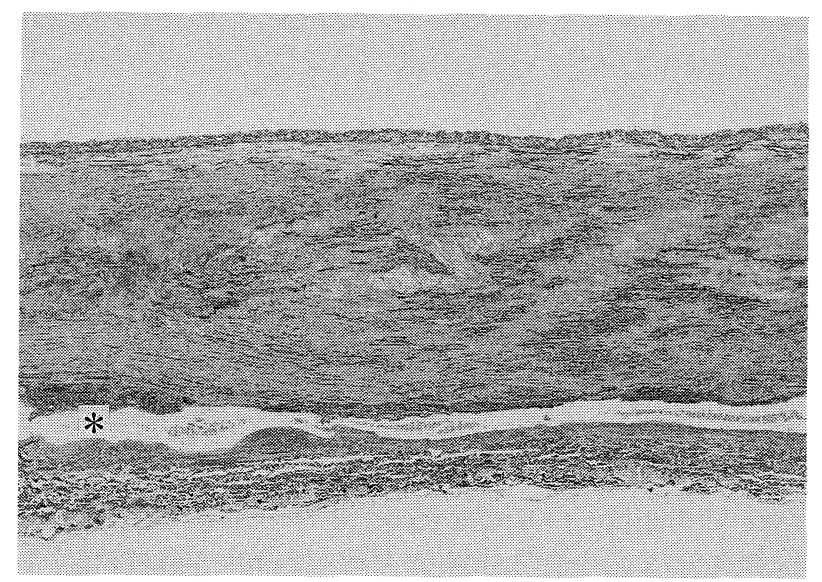

a

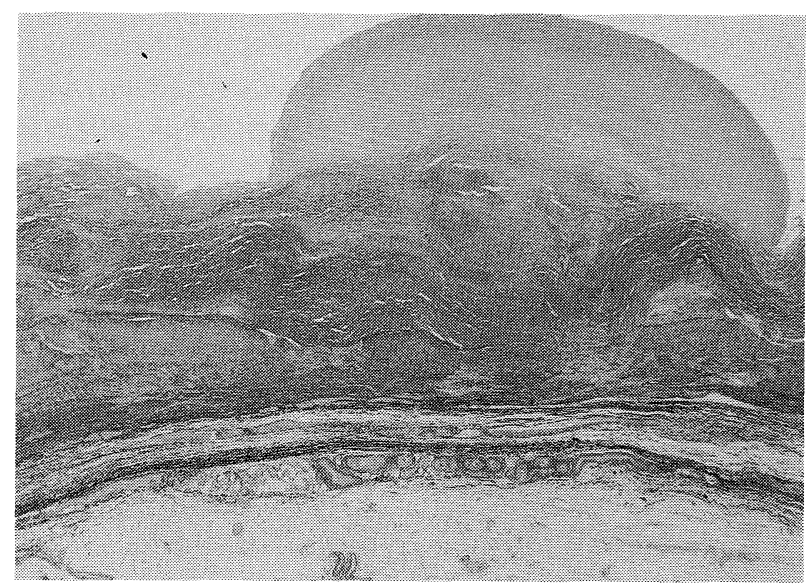

C thoracic aorta just distal to the ductus was $54 \mathrm{~mm}$, and that of the proximal segment of the abdominal aorta was $54 \mathrm{~mm}$. There was no obstructive narrowing in the major branches of the aorta.

Histologically, the medial layer of the main pulmonary artery showed marked disruption of the elastic layers and the accumulation of glycosaminoglycans. In the aneurysmal segment, the tunica media was ruptured (Fig. 3a). In the ascending aorta, no significant arteriosclerotic lesion was present, although the lamellar units of the tunica media were disturbed focally (Fig. 3b).

In the descending thoracic and abdominal aortas, lamellar fibroelastic thickening of the intima and adhesion of the fresh and organizing thrombi were frequently observed (Fig. $3 \mathrm{c}$ and $3 d)$. Fibrinogen was detected on the surface of these thickened lamellar structures (Fig. 4). A few cholesterol crystals and foam cells were observed in the area of the lamellar thickened intima.

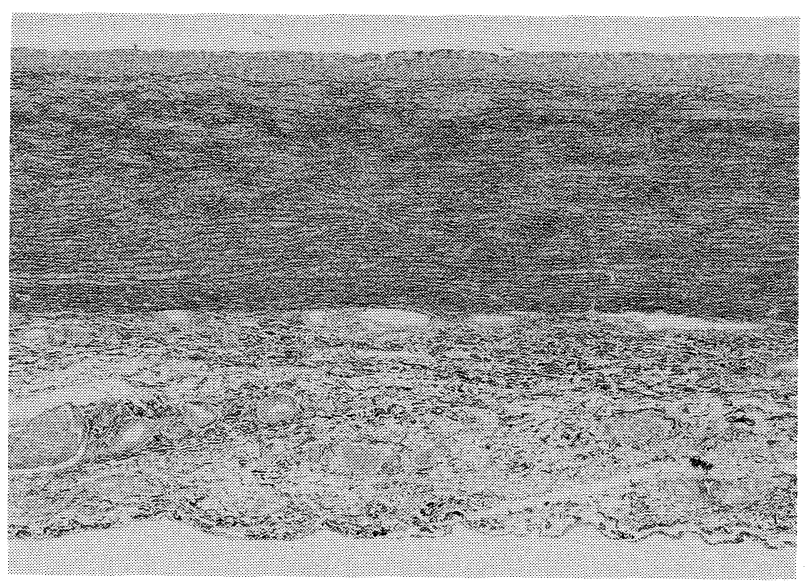

$\mathrm{b}$

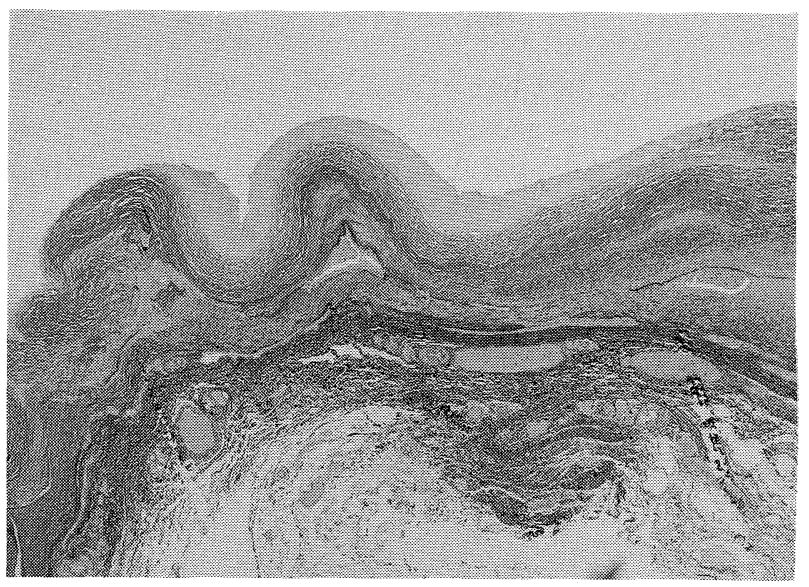

d

Figure 3. Microscopic findings of vessels. a) Pulmonary artery. The medial layer of the pulmonary artery adjacent to the rupture demonstrated marked elastin fragmentation and cystic medionecrosis. A tear is visible in the adventitia (*). There was no intimal thickening (Pentachrome stain, $\times \mathbf{4 0}$ ). b) Ascending aorta. The ascending aorta revealed slight intimal thickening and minimal fragmentation of the medial elastic tissue (Pentachrome stain, $\times 40)$. c) The descending thoracic aorta. Lamellar thickening is observable in the intima of the thoracic aorta. The uppermost layer of the intima consisted of amorphous material (organized thrombi). Vaso vasorum in the adventitia showed intimal thickening (Elastica-van Gieson stain, $\times 40$ ). d) The abdominal aorta. Organizing mural thrombi are located in the fibrously thickened intima (Pentachrome stain, $\times 20$ ). 


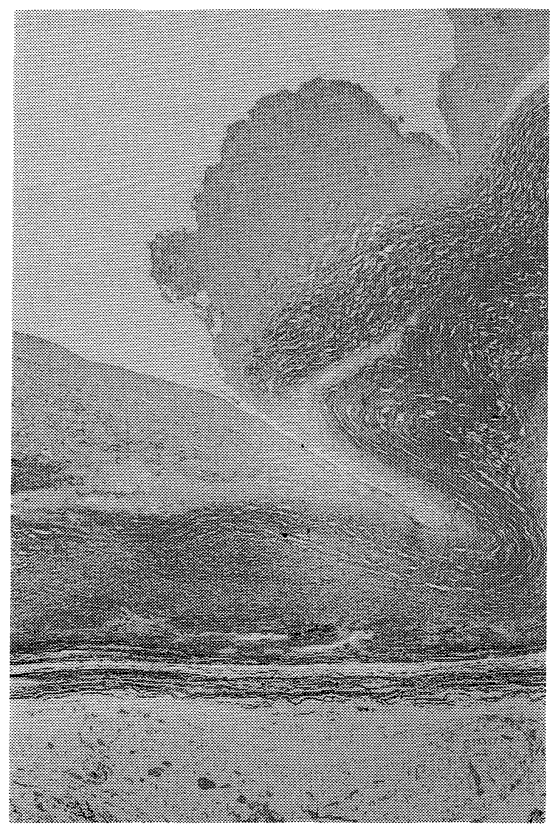

a

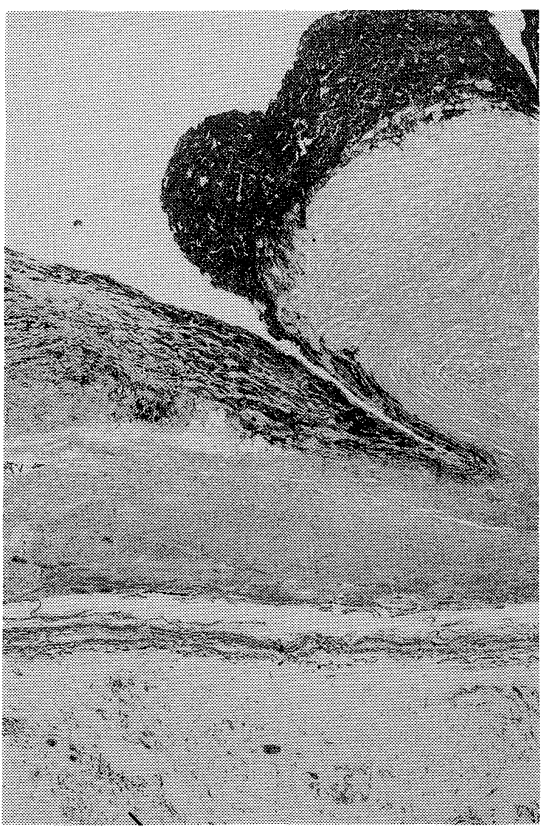

b

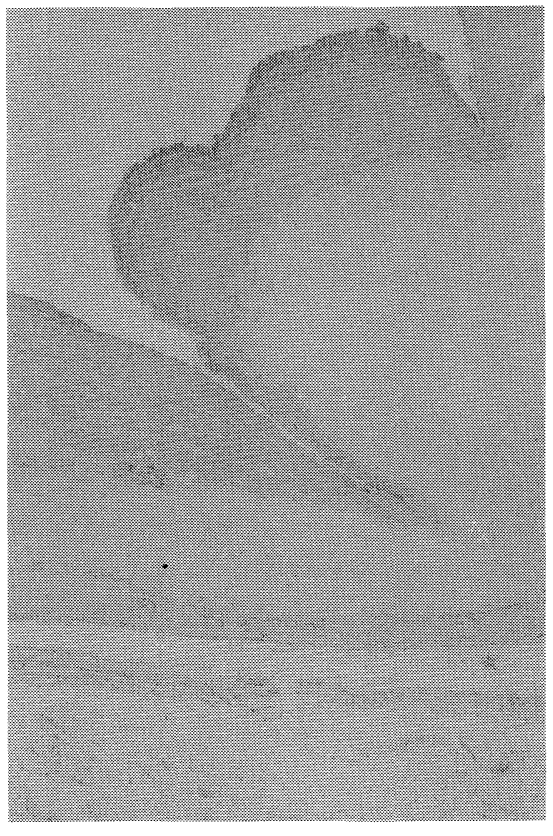

c

Figure 4. Organizing thrombus on the intima of the abdominal aorta. a) Fresh thrombus adhered to the intima, which consisted of the firm connective tissue. The media revealed marked structural injury (Elastica-van Gieson stain, $\times 20$ ). $b$ ) Phosphotungstic acid hematoxylin staining-positive material (fibrin) is observed in the fresh and organizing thrombus (Phosphotungstic acid hematoxylin stain, $\times \mathbf{2 0}$ ). c) Immunostaining of the thrombus. Positive staining for fibrinogen is noted in the upper layer of the fresh and organizing thrombi (Anti-human fibrinogen polyclonal antibody, $\times 20$ ).

The elastic layer and medial smooth muscles were also damaged.

\section{Discussion}

In this patient, the cause of death was rupture of the wall of a pulmonary artery aneurysm, which demonstrated severe cystic medial necrosis and elastin fragmentation attributable to the pulmonary hypertension (4-7). The most interesting finding in this case was the arteriosclerosis involving the descending thoracic and abdominal aortas. Although laboratory data on admission were incomplete because of the patient's sudden death, she had no cardiovascular risk factors for atherosclerosis. The lesion consisted of fibrous connective tissue and superimposing thrombi. Organizing process of the adhesive thrombi (8, 9) was frequently observed (Fig. 4). The atherosclerotic changes in the intima were minimal.

Several causative factors may have been involved in the development of the lesion: 1) sludging of blood due to erythrocytemia, 2) relatively low blood pressure, 3 ) a congenital vascular defect in the aorta, 4) focal hypercoagulability induced by turbulent flow through the ductus, and 5) hypoxia in the descending aorta.

In congenital heart disease in adolescents and adults, systemic hypercoagulability due to polycythemia frequently occurs, resulting in systemic thromboembolism (10). Thus, throm- bus formation in the descending thoracic aorta may have occurred in association with polycythemia vera (11).

In the present patient, the relative hypotension in the descending aorta may have caused changes in the vascular wall. The sludging of blood in the low-pressured aorta may have caused mural thrombi which produced an irregular, but otherwise diffuse, intimal thickening. However, this patient had no history of systemic thromboembolism. The possibility of turbulent flow can be excluded, because post-stenotic dilatation was absent in this patient. In patients with typical coarctation of the aorta, in which the descending aorta is constantly exposed to turbulent flow, arteriosclerotic changes are restricted to the ascending aorta and the area of poststenotic dilatation (12). Such lesions were not observed in the present patient. Although, it is well known that the abdominal aorta does not contain vasa vasorum, in contrast to the thoracic aorta (13), it is unlikely that a congenital defect of the media induced the intimal change, since the basic structure of the media was preserved in the ascending and descending thoracic aortas.

The major clinical differences between the ascending aorta and the descending aorta in this case were the blood pressure and oxygen content findings. The hypoxia may have directly injured the permeability of the endothelium (14). The injured intima lost its protective function against thrombus formation. Hypoxia has been considered as a major factor in experimental atherosclerosis of rabbits $(15,16)$. However, a relationship 


\section{Thrombosclerosis in Aortic Interruption}

between hypoxia and atherosclerosis in humans has not been demonstrated except for atherosclerosis related to smoking (17).

In conclusion, we hypothesized that the thrombosclerosis (18) of the descending aorta in this patient was caused by sludging of the blood and hemodynamic changes, in close association with erythrocytemia related to her condition.

Acknowledgements: The authors appreciate the advice of Professor Emeritus Kazuo Kitamura, and the invaluable contributions of Dr. Mitsuru Shimizu and Dr. Young Joon Lee.

\section{References}

1) Hammon JW Jr, Merrill WH, Prager RL, Graham TP Jr, Bender HW Jr. Repair of interrupted aortic arch and associated malformation in infancy: Indications for complete or partial repair. Ann Thorac Surg 42: 17, 1986.

2) Takashina $T$, Ishikura $Y$, Yamane $K$, et al. The congenital cardiovascular anomalies of the interruption of the aorta-Steidele's complex. Am Heart J 83: 93, 1972.

3) Freedom RM, Bain HH, Esplugas E, Dische R, Rowe RD. Ventricular septal defect in interruption of aortic arch. Am J Cardiol 39: 572, 1977.

4) Coleman M, Slater D, Bell R. Rupture of pulmonary artery aneurysm associated with persistent ductus arteriosus. Br Heart J 44: 464, 1980.

5) Nagelsmit MJ, Eulderink F. Dissecting aneurysm of the pulmonary trunk. Am J Cardiol 58: 660, 1986.

6) Yamamoto ME, Jones JW, McManus BM. Fatal dissection of the pulmonary trunk. An obscure consequence of chronic pulmonary hypertension. Am J Cardiovasc Pathol 1: 353, 1988.

7) Coard KCM, Martin MP. Ruptured saccular pulmonary artery aneurysm associated with persistent ductus arteriosus. Arch Pathol Lab Med 116: 159, 1992.

8) Prathap K. The morphology of two-year-old healed platelet-rich thrombi in femoral arteries of normocholesterolaemic monkeys: Light and electron-microscope observations. J Pathol 110: 145, 1973.

9) Stehbens WE. The role of thrombosis and variants of the thrombogenic theory in the etiology and pathogenesis of atherosclerosis. Prog Cardiovasc Dis 34: 325, 1992.

10) Perloff JK, Marelli AJ, Miner PD. Risk of stroke in adults with cyanotic congenital heart disease. Circulation 87: 1954, 1993 (comment).

11) Josephson GD, Tiefenbrun J, Harvey J. Thrombosis of the descending thoracic aorta: A case report. Surgery 114: 598, 1993.

12) Hudson REB. Cardiovascular Pathology. Vol. 2, Edward Arnord, Ltd., London, 1956, p. 2071.

13) Schlatmann TJM, Becker AE. Histologic changes in the normal and aging aorta: Implications for dissecting aortic aneurysm. Am J Cardiol 39: 13, 1977.

14) Astrup P, Kjeldsen $K$, Wanstrup J. The effect of exposure to carbon monoxide, hypoxia and hyperoxia on the development of experimental atheromatosis in rabbits. in: Atherosclerosis (Proceedings of the Second International Symposium), Jones RJ, Ed. Springer-Verlag, New York, Berlin, Heidelberg, 1970, p. 108.

15) Helin $P$, Lorenzen I. Arteriosclerosis in rabbit aorta induced by systemic hypoxia. Biochemical and morphological studies. Angiology 20: 1, 1969.

16) Okamoto R, Hatani M, Tsukitani M, et al. The effect of oxygen on the development of atherosclerosis in WHHL rabbits. Atherosclerosis 47:47, 1983.

17) Wald N, Howard S, Smith PG, Kjeldsen K. Association between atherosclerotic diseases and carboxyhaemoglobin levels in tobacco smokers. $\mathrm{Br}$ Med J 1: 761, 1973.

18) Moore $S$. Thromboatherosclerosis in normolipidemic rabbits. A result of continued endothelial damage. Lab Invest 29: 478, 1973. 